\title{
Le financement des cultes et la Convention européenne des droits de l'homme
}

\section{Gérard Gonzalez}

\section{OpenEdition}

\section{Journals}

Édition électronique

URL : http://journals.openedition.org/rdr/1022

DOI : $10.4000 /$ rdr.1022

ISSN : 2534-7462

\section{Éditeur}

Presses universitaires de Strasbourg

Édition imprimée

Date de publication : 10 mai 2016

Pagination : $9-21$

ISBN : 978-2-86820-954-2

ISSN : 2493-8637

\section{Référence électronique}

Gérard Gonzalez, "Le financement des cultes et la Convention européenne des droits de l'homme », Revue du droit des religions [En ligne], 1 | 2016, mis en ligne le 11 février 2020, consulté le 19 novembre 2020. URL : http://journals.openedition.org/rdr/1022 ; DOI : https://doi.org/10.4000/rdr.1022

\section{(c) (7) \&}

La revue du droit des religions est mise à disposition selon les termes de la Creative Commons Attribution - Pas d'Utilisation Commerciale 4.0 International - CC BY-NC 4.0. 


\section{LE FINANCEMENT DES CULTES ET LA CONVENTION EUROPÉENNE DES DROITS DE L'HOMME}

\section{Gérard GONZALEZ}

Université de Montpellier, Institut de droit européen des droits de l'homme (IDEDH)

\section{RÉSUMÉ}

La Cour européenne consacre la liberté des États de contribuer au financement des cultes. Elle accepte des différences de traitement entre les cultes dès lors que cette différence ne constitue pas une discrimination, c'est-à-dire qu'elle doit reposer sur des motifs objectifs et raisonnables, faute de quoi une discrimination pourrait être identifiée. L'arbitraire ne doit pas non plus être à l'origine d'une ingérence si grave et disproportionnée qu'elle remette en cause le fonctionnement du culte visé, portant ainsi une atteinte inconventionnelle à l'exercice collectif de la liberté de religion, mais aussi, au-delà, à l'exercice individuel de chacun des fidèles du groupe concerné. Sur ce terrain du financement des cultes, le principe de subsidiarité trouve une application satisfaisante.

\section{ABSTRACT}

The European Court enshrines freedom of States to finance religious groups. A difference in treatment will be acceptable if it is not discriminatory, that is based on objective and reasonable grounds. The arbitrariness should not be the cause of so serious and disproportionate interference that call into question the operation of religious groups involved, bringing a disproportionate interference with the collective exercise of freedom of religion, but also beyond, to the individual freedom of religion of the faithful. On this ground of religion funding, there is a satisfactory application of the subsidiarity principle. 
Toute religion a, pour son fonctionnement besoin de financement.

1 Celui-ci est variable selon les relations qu'entretiennent (ou pas) les États avec les religions présentes sur leur sol. Au sein des quarante-sept États membres du Conseil de l'Europe, et donc parties à la Convention européenne des droits de l'homme, ces relations sont très diverses, de la laïcité prônant la séparation jusqu'au système d'Église d'État. Cette diversité n'est pas remise en question par l'adhésion à la Convention. La Commission, comme la Cour, ont adopté la seule attitude possible en se refusant à condamner a priori tout système de relations entre l'État et un (ou des) culte(s), la Commission jugeant par exemple qu' "en soi », le système d'État confessionnel ne viole pas l'article 9 de la Convention ${ }^{1}$, principe de neutralité de la Convention à l'égard des systèmes nationaux que la Cour n'a jamais remis en question, sauf au regard de l'État fondé sur une véritable théocratie ${ }^{2}$. La question du financement des cultes se présente dès lors devant la Cour européenne des droits de l'homme sous diverses formes. Certaines ne concernent pas directement l'exercice d'un culte dans le cadre de la liberté de religion. La jurisprudence européenne écarte toute prétention dont le lien avec l'objet cultuel apparaît trop peu évident ou inexistant. Ainsi, l'Église de Scientologie se plaignaitelle d'une ingérence injustifiée dans l'exercice de son droit à exprimer une opinion religieuse à propos d'une annonce proposant à la vente l'électromètre Hubbard (E-mètre) dont la publicité avait été censurée en ce qu'elle le présentait comme "un moyen précieux de mesurer l'état de l'âme humaine et ses variations ». Or l'article 9 « ne protège pas des professions de prétendue foi religieuse qui apparaissent comme des arguments de vente dans des annonces à caractère purement commercial faites par un groupe religieux ${ }^{3}$. D'autres voies sont cependant plus ouvertes, comme à tout particulier, qu'il s'agisse de la protection du droit de propriété également protégé par la Convention selon l'article 1 Protocole 1 (1P1) ou de l'article 6 garantissant le droit à un procès équitable. L'illustre l'affaire Les Saints monastères c/ Grèce dans laquelle des entités légales de droit public se virent privées de certaines possessions dont la preuve d'un droit de propriété n'avait pu, compte tenu de leur ancienneté, être rapportée comme l'exigeait une nouvelle $\operatorname{loi}^{4}$. La Commission décide que « les dispositions incriminées se réfèrent au patrimoine monastique et

1. Rapport 9 mai 1989, n 11581/85, Peter Darby c/ Suède.

2. CEDH, Gde ch., 13 févr. 2003, Refah Partisi et a. c/ Turquie, obs. Gonzalez G., in Sudre F. (dir.), Les grands arrêts de la Cour européenne des droits de l'homme, Paris, PUF, 7 éd. 2015, $\mathrm{n}^{\circ} 57$.

3. Comm. EDH, déc. 5 mai 1979, n 7805/77, X. et Église de scientologie c/ Suède.

4. CEDH, 9 déc. 1994, n 13092/87; 13984/88. 
ne concernent nullement la pratique religieuse de l'ascétisme » et que « les requérants n'ont pas démontré que d'autres objets ou avoirs nécessaires à la pratique et au culte religieux étaient affectés ». Elle observe que « les zones qui entourent les monastères et, en particulier, celles destinées à la culture par les moines, sont exemptées des dispositions incriminées ». Analyse sévère et minimaliste que la Cour est venue un peu tempérer en relevant une violation du droit au respect des biens de certains des requérants, tout en précisant que « les dispositions jugées contraires à l'article $1 \mathrm{P} 1$ ne visent en aucune manière les biens des requérants destinés à la pratique du culte et, partant, ne portent pas atteinte à l'exercice du droit à la liberté de religion ». La Cour européenne accorde aux Saints Monastères une protection identique à celle des particuliers touchés par des procédures d'expropriation, en faisant porter son contrôle sur le respect de l'article 1P1 relatif au respect des biens. Parfois cependant, l'atteinte aux biens est telle, si récurrente et grave, que le constat de violation de l'article 1P1 englobe implicitement une violation discriminatoire de la liberté de religion que la Cour ne constate pas expressément par économie de moyens, comme dans le cas des affaires relatives aux minorités et fondations de droit turc ${ }^{5}$. La question qui sera abordée ici le sera plus précisément sous l'angle du financement des cultes contribuant directement à l'exercice de la liberté de religion, dont le culte est la manifestation collective essentielle. Dans ce cadre, la Convention n'interférant pas avec le choix, souvent culturel et historique, de l'État de soutenir les cultes, le principe de la liberté de celui-ci d'accorder un soutien financier semble acquis sous certaines conditions (1). Par ailleurs, soucieuse de garantir des droits « concrets et effectifs», la Cour veille au respect par l'État de l'autonomie ecclésiale au regard de son propre financement (2).

\section{LE PRINCIPE DE LA LIBERTÉ DE L'ÉTAT POUR ACCORDER UN SOUTIEN FINANCIER CULTUEL}

L'indifférence de la Convention aux types de relations Églises-État, exception faite de la théocratie, suscite néanmoins un encadrement des liens tressés entre un État et une religion en particulier. Dès lors se pose la question de l'impôt ecclésial (1.1) et du respect de l'interdiction des discriminations

5. CEDH, 9 janv. 2007, n 34478/97, Fener Rum Erkek Lisesi Vakfı c/ Turquie; 8 juill. 2008, $\mathrm{n}^{\circ}$ 14340/05, Fener Rum Patrikliği (Patriarcat oecuménique) c/ Turquie; 3 mars 2009, $\mathrm{n}^{\circ} 37639 / 03$ et autres, Bozcaada Kimisis Teodoku Rum ortodoks Kilisesi Vakfi c/ Turquie; 6 oct. 2009, n 35570/02, Özbek c/ Turquie. 
fondées sur la religion qui pourraient résulter d'un soutien financier trop exclusif d'une religion (1.2).

\subsection{LA CONVENTIONNALITÉ SOUS RÉSERVE DE L'IMPÔT ECCLÉSIAL}

\subsubsection{Le principe de la conventionnalité de l'impôt ecclésial}

Dans l'affaire Darby, la question de la validité de cet impôt ne fut pas soulevée, mais la Cour conclut à un refus discriminatoire d'exonération du requérant fondée sur sa résidence et ne reposant sur aucun but légitime ${ }^{6}$. Implicitement, c'était accepter le principe d'un tel impôt collecté au profit de l'Église luthérienne de Suède dont une partie seulement (30\%) était payée par les personnes n'appartenant pas à cette Église pour la couverture des tâches administratives remplies par les paroisses. De telles activités « civiles » peuvent justifier l'attribution d'un financement spécifique aux Églises concernées, en principe l'Église d'État, sans que cela emporte « en soi » violation de la Convention $^{7}$. La Cour va pouvoir livrer plus ouvertement sa position sur la conventionnalité de l'impôt ecclésial dans sa décision d'irrecevabilité d'une plainte contestant le fait que, dans le cadre de leur déclaration de revenus, les requérants espagnols ne pouvaient, en tant que membres de l'Église évangélique baptiste, affecter directement une partie de leur impôt au soutien de leur Église, à la différence des contribuables de religion catholique ${ }^{8}$. La Cour confirme que « la liberté de religion n'implique nullement que les Églises ou leurs fidèles doivent se voir accorder un statut fiscal différent de celui des autres contribuables » et que les États jouissent dans ce domaine d'une « marge d'appréciation d'autant plus justifiée qu'il n'existe pas au niveau européen un standard commun en matière de financement des Églises ou cultes; ces questions étant étroitement liées à l'histoire et aux traditions de chaque pays ». Par cette décision, la Cour valide le système du concordat, observant que le « traitement fiscal spécifique dont bénéficie l'Église catholique en Espagne [...] découle des accords conclus le 3 janvier 1979 entre l'Espagne et le Saint-Siège, qui mettent à la charge des deux parties des obligations réciproques : par exemple, l'Église catholique s'engage à mettre au service de la société son patrimoine historique, artistique et documentaire »; en contrepartie "ses locaux de culte bénéficient d'une exonération fiscale».

6. CEDH, 23 oct. 1990, n 11581/85, Darby c/ Suède.

7. CEDH, déc. 18 sept. 2012, n 22897/08, Asatruarfelagio c/ Islande.

8. CEDH, déc. 14 juin 2001, n53072/99, Alujer Fernandez et Rosa Caballero Garcia c/ Espagne. 
D’une façon générale, « l'obligation faite à une Église de conclure un accord avec l'État afin de pouvoir bénéficier de la rétrocession d'une partie de l'impôt sur le revenu recouvré par l'État n'apparaît pas comme étant une exigence dénuée de fondement ni disproportionnée "; toutefois, la Cour fixe une limite aux conséquences de tels accords qui ne doivent pas constituer un avantage discriminatoire ${ }^{9}$.

\subsubsection{Les modalités de gestion de l'impôt ecclésial}

Selon une loi italienne de 1985, huit millièmes de l'impôt sur le revenu sont destinés à l'État, à l'Église catholique ou à l'une des institutions représentatives des cinq autres religions (Union des Églises adventistes du septième jour, Assemblée de Dieu d'Italie, Église méthodiste et vaudoise, Église évangélique luthérienne et Union des communautés juives d'Italie) qui ont conclu avec l'État une convention. Le choix de la destination de ce pourcentage d'impôt doit être exprimé lors de la déclaration de revenus et si aucun choix n'est exprimé, la somme est partagée entre l'État, l'Église catholique et les institutions représentatives des autres religions. Le requérant se plaint d'être obligé de manifester ses convictions lors de la rédaction de sa déclaration de revenus. Lapidairement, la Cour déclare la requête irrecevable sur le terrain de l'article 9 de la Convention au motif que « les contribuables ont la faculté de ne pas exprimer de choix quant à la destination des huit millièmes de l'impôt sur le revenu », d'où « il s'ensuit que la disposition litigieuse n'entraîne pas une obligation de manifester ses convictions religieuses pouvant être considérée comme contraire aux articles de la Convention invoqués». Sur le terrain du droit des biens, tout aussi abruptement, la Cour juge l'argument irrecevable car « la loi fiscale litigieuse prévoit non pas une imposition qui s'ajouterait à l'impôt normal sur le revenu, mais seulement une affectation spécifique d'un pourcentage des sommes perçues au titre de cet impôt », cette loi s'inscrivant dans la marge d'appréciation de l'État « ne saurait être considérée en tant que telle comme arbitraire $»^{10}$. L'affaire Wasmuth $\mathrm{c}$ Allemagne ${ }^{11}$ va lui donner, quelques mois plus tard, l'occasion de livrer au fond une motivation plus précise, faute d'être complètement convaincante. En Allemagne, les contribuables disposent d'une carte d'imposition sur le salaire assortie d'une rubrique concernant le prélèvement de l'impôt cultuel retenu et versé au Trésor public par les employeurs. Sur la carte d'imposition du requérant,

9. V. 1.2.

10. CEDH, déc. 29 mars 2007, n²3123/04, Spampinato c/ Italie.

11. CEDH, 17 févr. 2011, nº 12884/03, Wasmuth c/ Allemagne. 
cette rubrique contient la mention «-- », indiquant sa non-appartenance à une Église ou une société religieuse habilitée et informant son employeur qu'il n'y a pas lieu de retenir cet impôt. Selon le requérant, l'obligation qui lui est faite d'indiquer son appartenance religieuse enfreint l'article 9 de la Convention. Logiquement, la Cour retient « une ingérence dans le droit du requérant de ne pas déclarer ses convictions religieuses » (\$51). Pour démarquer son raisonnement de l'affaire ayant entraîné la condamnation de la Turquie pour la présence, même non renseignée, d'une case portant la mention « religion » sur les cartes d'identité ${ }^{12}$, la Cour juge qu'en l'espèce le requérant ne souhaite pas faire valoir ses droits contre les intérêts des autorités étatiques, mais que « les juridictions allemandes étaient appelées en l'espèce à mettre en balance l'aspect négatif de la liberté religieuse du requérant avec le droit des Églises et des sociétés religieuses de lever l'impôt cultuel », en tenant compte de l'obligation de l'État de garantir le prélèvement en bon ordre de cet impôt. Autrement dit, il s'agit ici d'un contentieux de nature non pas verticale mais horizontale nécessitant de résoudre un conflit de droits. Selon la Cour, « la mention « -- » sur la carte d'imposition n'a qu'une portée limitée en ce qui concerne l'appartenance et l'orientation religieuse ou philosophique du requérant [...] [Elle] renseigne les autorités fiscales uniquement sur le fait que le requérant n'appartient pas à l'une des six Églises ou sociétés religieuses habilitées à lever l'impôt cultuel »(\$58). De plus, « les autorités nationales n’ont pas demandé au requérant d'exposer pourquoi il n'appartenait pas à l'une des Églises ou sociétés religieuses levant l'impôt cultuel et n'ont pas vérifié quelle était son orientation religieuse ou philosophique »; l'ingérence n'est donc pas disproportionnée (860-61). C'est faire peu de cas de l'aspect négatif de la liberté de manifester sa religion qui comporte le droit de ne pas être obligé d'agir de telle sorte que l'État ou l'employeur puisse en déduire que l'on a ou pas de telles convictions.

\subsection{L'INTERDICTION D'AVANTAGES DISCRIMINATOIRES}

L'État peut prévoir des régimes juridiques différents comme l'ont admis la Commission et la Cour, mais dans la jouissance des droits qui en découlent, il lui appartient de veiller à ce que cette différence de traitement soit dictée par des justifications objectives et raisonnables. Ainsi la Commission a-t-elle eu l'occasion de se prononcer sur la distinction opérée en droit français entre les associations de droit commun et les associations cultuelles de la loi de 1905

12. CEDH, 2 févr. 2010, n² 21924/05, Sinan Isık c/ Turquie. 
à la requête de l'Union des athées. La Commission note que l'une des principales distinctions « réside dans la possibilité, accordée aux unes, refusée aux autres, de recevoir à titre gratuit ». Insatisfaite de la seule justification tenant au risque de captation d'héritage, la Commission ne retient « aucune justification objective et raisonnable de maintenir un tel système qui défavorise à un tel degré les associations non cultuelles ${ }^{13}$. La Cour n'a pas été saisie de cette affaire. Dans l'affaire Alujer Fernande $z^{14}$, la Cour ayant validé le principe de l'impôt ecclésial résultant d'un concordat a aussi fixé les limites de cette situation privilégiée. Selon elle, « la conclusion d'accords entre un État et une Église donnée prévoyant un statut fiscal spécifique en sa faveur ne s'oppose pas, en principe, aux exigences découlant des articles 9 et 14 de la Convention dès lors que la différence de traitement s'appuie sur une justification objective et raisonnable et qu'il est possible de conclure des accords similaires avec d'autres Églises qui en exprimeraient le souhait ", souhait que l'Église des requérants n'a pas formulé. Le principe est donc qu'une Église sans accord puisse entamer des démarches en vue d'en conclure un, sans pour autant que le résultat positif soit assuré. En effet, au regard des contreparties apportées par l'Église catholique à l'État (voir supra), il semble difficile d'imposer à l'État de conclure un accord avec une Église qui n'en offrirait aucune et, du point de vue culturel et historique, aucune Église implantée en Espagne n'est susceptible de rivaliser avec l'Église catholique, ce qui pourrait légitimer implicitement son statut exclusif. En l'espèce, le traitement spécifique dont bénéficie l'Église catholique est d'autant plus acceptable que « la législation fiscale espagnole ne contraint personne à [lui] verser une partie de ses impôts », chaque contribuable pouvant destiner le pourcentage de ses impôts fixé par la loi budgétaire à des fins d'intérêt social ou général, la législation espagnole permettant par ailleurs à toute personne d'effectuer « des dons à l'Église de son choix dans des conditions fiscales avantageuses ». La Commission avait déjà appliqué les mêmes principes en ce qui concerne l'exonération de taxe foncière dont bénéficie en Espagne la seule Église catholique en vertu du même accord ${ }^{15}$. Par ailleurs, la Cour dresse un constat de non-violation à l'égard d'une législation établissant une exonération de la taxe foncière des entreprises au bénéfice des seuls lieux de culte ouverts au public, cette législation étant appliquée indistinctement et l'Église requérante, dont le temple n'est pas ouvert au public, bénéficiant, en tant qu'organisme caritatif, d'un

13. Rapport 6 juill. 1994, n 14635/89, Union des athées c/ France.

14. Op. cit.

15. Comm. EDH, déc. 11 janv. 1992, n 17522/90, Iglesia Bautista «El Salvador» et José Aquilino Ortega Moratilla c/ Espagne. 
abattement substantiel ${ }^{16}$. En revanche, le refus de considérer les cemevi, lieux de réunions cultuelles des alévis, comme des lieux de cultes reconnus leur interdisant de bénéficier de la prise en charge des frais d'électricité par l'État comme les autres lieux de culte reconnus constitue une discrimination fondée sur la religion ${ }^{17}$. Ce souci de la Cour de ne pas entraver les relations spécifiques d'un État avec une ou plusieurs religions se double de celui tout aussi fort de ne pas laisser ce type de relations hors de contrôle et de préserver l'autonomie financière de tous les cultes.

\section{LES OBLIGATIONS DE RESPECT DE L'AUTONOMIE FINANCIÈRE DES CULTES}

Le principe de l'autonomie ecclésiale est aujourd'hui bien établi par la jurisprudence de la Cour européenne selon laquelle « le droit des fidèles à la liberté de religion suppose que la communauté puisse fonctionner paisiblement, sans ingérence arbitraire de l'État » et « l'autonomie des communautés religieuses est indispensable au pluralisme dans une société démocratique et se trouve au cœur même de la protection offerte par l'article 9 de la Convention ${ }^{18}$. Cette autonomie passe indéniablement par l'attribution d'un statut juridique minimal par l'État (2.1) et par une retenue des ingérences étatiques attentatoires aux ressources des Églises (2.2).

\subsection{L'OBLIGATION POSITIVE DE CONFÉRER UN STATUT JURIDIQUE}

Il peut exister comme démontré ci-dessus des régimes juridiques différents pour les cultes, pour autant que ces différences n'entraînent pas de discriminations susceptibles de porter atteinte à l'exercice de la liberté de religion notamment des fidèles. Parfois le problème est plus structurel encore, une Église ne pouvant exercer convenablement son droit à la liberté d'association interprété à la lumière des principes de la liberté de religion. Ainsi, l'exercice collectif de la liberté de religion pose le problème du respect de la liberté d'association garanti par l'article 11 de la Convention. Confrontées à

16. CEDH, 4 mars 2014, n 7552/09, Church of Jesus Christ of Latter-Day Saints c/ Royaume-Uni.

17. CEDH, 2 déc. 2014, n 32093/10, Cumhuriyetçi Eğitim Ve Kültür Merkezi Vakfı c/ Turquie.

18. CEDH, Gde ch., 9 juill. 2013, n 2330/09, Sindicatul «Păstorul cel Bun » c/ Roumanie, $\S 136$; V. GonZalez G., " Lautonomie ecclésiale au risque relatif des droits de l'homme ", RTDH 2014, p. 803-818. 
un problème d'accès à un statut juridique déterminé, de différence de traitement sur la base de statuts juridiques différents, la Commission et la Cour seront amenées le plus souvent à privilégier l'examen de la requête sous cet angle plutôt que celui de l'article 9 isolément ou combiné avec l'article 14. Le principe clairement posé par la jurisprudence est que tout groupement religieux doit être en mesure d'accéder à un statut qui lui donne, au moins, la possibilité de défendre ses intérêts, notamment sa liberté de religion, son droit de propriété, devant les tribunaux ${ }^{19}$. La question structurelle concerne indirectement le financement des cultes (défense du droit de propriété, statut fiscal), il peut aussi le mettre en question plus directement et, en cas de dissolution d'une entité religieuse, la Cour liste une série de conséquences négatives notamment sur « le droit de posséder ou de louer la propriété, de détenir des comptes bancaires, d'embaucher des employés et de veiller à la protection judiciaire de la communauté, ses membres et ses actifs ${ }^{20}$. Dans une affaire récente, la Cour s'est prononcée sur l'allocation de subventions publiques à des Églises considérées comme « légalement établies » en vertu d'une loi nouvelle, alors que d'autres, légalement enregistrées auparavant, ne se virent pas reconnaître comme telles et ne jouirent plus en conséquence des mêmes avantages pécuniaires et fiscaux ${ }^{21}$. Pour ces dernières, une demande d'enregistrement devait être adressée qui ne recevrait une réponse favorable qu'en fonction de critères tels le nombre de membres, l'ancienneté de l'existence et la preuve qu'elles ne représentaient pas un danger pour la démocratie. La Cour rappelle que « la possibilité pour les citoyens de former une personne morale afin d'agir collectivement dans un domaine d'intérêt commun constitue un des aspects les plus importants du droit à la liberté d'association, sans quoi ce droit se trouverait dépourvu de toute signification » et « le refus des autorités internes d'accorder la personnalité juridique à une association de personnes privées s'analys[e] en une ingérence dans l'exercice de leur droit à la liberté d'association » (\$78). S'agissant de l'obligation positive « de mettre en place un système de reconnaissance facilitant l'acquisition de la personnalité juridique par les communautés religieuses », la Cour rappelle que « le devoir de neutralité et d'impartialité de l'État [...] est incompatible avec un quelconque pouvoir d'appréciation de la part de l'État quant à la légitimité des croyances religieuses » (\$90). Puis la Cour souligne que « les différences entre

19. CEDH, 13 déc. 2001, n 45701/99, Église métropolitaine de Bessarabie et a. c/ Moldavie.

20. CEDH, 12 juin 2014, $n^{\circ}$ 33203/08, Biblical Centre of the Chuvash Republic v. Russia, $\$ 60$ (l'arrêt n'existe qu'en anglais - traduit par nous).

21. CEDH, 8 avril 2014, n 70945/11 et autres, Magyar Keresztény Mennonita Egyház et autres c/ Hongrie. 
les statuts juridiques accordés aux communautés religieuses ne doivent pas donner de leurs adhérents une image défavorable aux yeux du grand public [...] Dans les traditions de nombreux pays, la désignation en tant qu'Église et la reconnaissance de l'État sont les clés du statut social, en l'absence duquel la communauté religieuse peut être vue comme une secte douteuse. En d'autres termes, le refus de reconnaître une communauté religieuse en tant qu'Église peut amplifier les préjugés contre les adhérents de telles communautés, souvent de petite taille, particulièrement dans le cas des religions professant des enseignements nouveaux ou inhabituels »(\$92). Il s'agit là sans doute de la synthèse la plus marquante de la défiance qu'a toujours manifestée la Cour à l'égard de la labellisation négative de « secte ${ }^{22}$. Selon la Cour, « ces différences [dans le statut juridique et le traitement qui en résulte entre les communautés religieuses en termes de coopération avec l'État] ont un impact sur l'organisation de la communauté et donc sur la pratique, individuelle ou collective, de la religion"; dès lors « la Cour ne peut ignorer le risque que les adhérents d'une religion puissent avoir l'impression de n'être que tolérés - mais pas bienvenus - si l'État refuse de reconnaître et soutenir leur organisation religieuse tout en accordant ce bénéfice à d'autres institutions »(\$93-94). Les distinctions ainsi opérées en matière de partenariat et de subventions ne doivent pas produire « une situation dans laquelle les adhérents d'une communauté religieuse auraient l'impression d'être des citoyens de seconde zone, pour des raisons religieuses, en raison d'une position de l'État moins favorable envers leur communauté » (\$109). En l'espèce, le droit hongrois fait bénéficier les Églises reconnues d'un traitement préférentiel, « en particulier dans le domaine de la fiscalité et des subventions » et les avantages obtenus sont substantiels et « les aident à assumer des activités religieuses en raison de leur forme organisationnelle spécifique » (\$110). Parmi les privilèges liés à l'enregistrement, seules les Églises « incorporées » ont droit à un pour cent de l'impôt sur le revenu des particuliers déclaré par les croyants et à la subvention correspondante de l'État. Ces sommes étant destinées à « soutenir les activités confessionnelles », la Cour estime que cette différenciation ne satisfait pas aux exigences de «neutralité de l'État et [...] impose un fardeau aux croyants de petites communautés religieuses sans raison objective et valable» (\$112). Ce défaut structurel, déconnecté d'un contexte culturel et historique semblable à celui de l'affaire Alujer Fernandez par exemple, nuit non seulement aux

22. CEDH, 6 nov. 2008, Leela Förderkreis E.V. cl Allemagne, RTDH 2009, p. 553-568, obs. GonZalez G. ; CEDH, 10 juin 2010, Témoins de Jéhovah de Moscou c/ Russie, RTDH 2011, p. 199-217, obs. GONZALEZ G. 
finances du culte concerné mais, et ce lien est établi aussi clairement pour la première fois, sur l'image du culte en question et de ses adeptes.

\subsection{L'ENCADREMENT DES INGÉRENCES ÉTATIQUES ATTENTATOIRES AUX RESSOURCES CULTUELLES}

Déjà dans l'affaire des Saints monastères la Cour avait souligné l'importance, pour apprécier l'ingérence d'un État dans la liberté de religion par le biais d'une atteinte aux biens, de vérifier que les « objets ou avoirs nécessaires à la pratique et au culte » n'étaient pas concernés. La question s'est posée de façon directe dans le cadre du contentieux opposant la France à l'association des Témoins de Jéhovah pour la taxation des dons manuels faits par les fidèles. Par son arrêt du 30 juin 2011, la Cour européenne conclut à la violation de l'article 9 de la Convention pour imprévisibilité de la « loi » française, c'est-àdire de l'interprétation totalement nouvelle et inattendue de l'article 757 du Code général des impôts instituant une taxation à hauteur de $60 \%$ des dons manuels « révélés » ${ }^{23}$. À l'évidence, une taxation de $60 \%$ des dons de fidèles à une association religieuse augmentée de pénalités qui, cumulées, dépassent les 50 millions d'euros constitue bien une ingérence dans la liberté de religion de l'association. Au gouvernement qui plaidait que « la taxation des dons manuels n'a eu aucun effet sur la liberté de religion de la requérante et de celle de ses membres, demeurés libres de pratiquer leur culte comme ils l'entendent », la Cour répond « que les dons litigieux constituant la source essentielle de financement de l'association par les fidèles, ceux-ci peuvent prétendre être directement affectés par la mesure fiscale [...] la taxation dont il s'agit a menacé la pérennité, sinon entravé sérieusement l'organisation interne, le fonctionnement de l'association et ses activités religieuses, étant observé que les lieux de culte étaient eux-mêmes visés » (\$53). L’ingérence est donc bien avérée. Qu'il s'agisse de la notion de "dons manuels », de l'identification du « donataire », de celle de "révélation » des dons, la Cour juge que l'association n'était pas «à même de prévoir à un degré raisonnable les conséquences pouvant résulter de la perception des offrandes et de la présentation de sa comptabilité à l'administration fiscale » (\$72). En l'espèce, la brutalité du revirement, notamment quant à l'interprétation de la notion de "révélation », ses conséquences financières exorbitantes pour l'association requérante et, indirectement, la communauté de ses membres, enfin son bénéfice limité pour le "progrès interprétatif » portent atteinte à la substance des principes de 
sécurité juridique et de confiance légitime. La Cour n'est « pas convaincue que la requérante était à même de prévoir à un degré raisonnable les conséquences pouvant résulter de la perception des offrandes et de la présentation de sa comptabilité à l'administration fiscale » (\$72). L'affirmation de l'imprévisibilité de la loi est si abrupte que l'on se demande comment les juridictions nationales ont pu avaliser l'instrumentalisation du contrôle fiscal effectué au siège de la requérante qui relève manifestement d'un manque de "cohérence » et de « loyauté » des autorités administratives. La violation de l'article 9 de la Convention étant identifiée dès le premier stade du contrôle de la loi, la Cour « n'estime pas nécessaire de se pencher sur le respect des autres exigences de l'article $9 \$ 2 »$, la finalité et la nécessité de l'ingérence (\$72). Avec le Président Costa on peut regretter cette économie, "parce que certaines des questions soulevées par la requête, si elles avaient dû être tranchées, auraient revêtu [...] un caractère délicat, et peut-être même "grave" au sens de l'article 30 de la Convention » (opinion séparée). L'instrumentalisation de la législation fiscale utilisée pour restreindre le pluralisme religieux de façon « arbitraire » n'apparaît pas nécessaire dans une société démocratique lorsqu'elle aboutit à des conséquences aussi radicales que la saisie de l'ensemble des biens de l'association concernée, notamment des lieux de culte, mettant en cause l'existence et la pérennité de l'organisation ayant en charge la liberté collective de religion des fidèles. La Cour appliquera le même raisonnement dans trois autres affaires identiques concernant de nouveaux mouvements religieux ${ }^{24}$. Sème en revanche la confusion sur la conventionnalité du principe d'une telle taxation la déclaration d'irrecevabilité de la plainte d'un quatrième groupement pour qui la taxation des dons manuels à hauteur de $60 \%$ n'avait pas, selon la Cour, pour conséquence de « couper ses ressources vitales ni d'entraver son activité religieuse ${ }^{25}$.

Vu de la Cour européenne des droits de l'homme, les États sont libres de contribuer au financement des cultes; des différences de traitement entre les cultes sont possibles sous réserve d'être justifiées par des motifs objectifs et

24. CEDH, 31 janv. 2013, $n^{\circ}$ 50471/07, Association Cultuelle du Temple Pyramide c/ France; 31 janv. 2013, n 50615/07, Association des Chevaliers du Lotus d'Or c/ France; 31 janv. 2013, n 25502/07, Église évangélique missionnaire et Salaûn c/ France.

25. CEDH, déc. 8 janvier 2013, n 41729/09, Sukyo Mahikari France c/ France: confusion regrettable, la Cour n'évoquant même pas dans cette décision d'irrecevabilité l'imprévisibilité de la loi sévèrement fustigée dans les quatre autres affaires ; y aurait-il deux poids deux mesures au sein même des groupements minoritaires? Dans aucune des cinq affaires concernant la taxation des dons manuels en France, la question d'une discrimination fondée sur la religion n'est examinée, soit par économie de moyens, soit parce que l'argument est jugé irrecevable. 
non arbitraires, faute de quoi une discrimination pourrait être identifiée. La Cour européenne, soucieuse du principe de subsidiarité, respecte les choix étatiques découlant d'un contexte social, historique particulier comme l'a illustré l'affaire Lautsi c/ Italie. L'arbitraire ne doit pas être le moteur du soutien accordé à une religion en particulier, il ne doit pas non plus être celui d'une ingérence si grave et disproportionnée qu'elle remette en cause le fonctionnement du culte visé, portant ainsi une atteinte disproportionnée à l'exercice collectif de la liberté de religion mais aussi, au-delà, à l'exercice individuel de chacun des fidèles du groupe concerné. Finalement, dans ce domaine délicat du financement des cultes, la Cour européenne semble avoir trouvé le juste milieu qui sied au respect raisonnable du principe de subsidiarité. 